\title{
NOUVELLE
}

\section{Synthèse des peptides antigéniques du CMH-I dans le noyau}

\section{Enfin un rôle pour la traduction nucléaire?}

Emilie Duvallet, Mathilde Boulpicante, Sébastien Apcher
Institut Gustave Roussy, Université Paris Sud, département d'immunologie, Unité 1015 , 114, rue Édouard Vaillant, 94805 Villejuif, France. sebastien.apcher@inserm.fr
> La quasi-totalité des études menées depuis une vingtaine d'années sur l'approvisionnement et l'apprêtement des peptides antigéniques au complexe majeur d'histocompatibilité de classe I (CMH-I) montrent que la source majeure de ces peptides provient de la dégradation de protéines endogènes. Cette dégradation se fait via le complexe du protéasome, système protéolytique majeur du cytosol [1]. C'est en 1996 que tous ces modèles vont être remis en cause par l'équipe de Yewdell et al., qui fut la première à émettre l'hypothèse selon laquelle les peptides présentés par les molécules du CMH-I ne proviendraient pas de la dégradation de protéines entières, mais plutôt de produits défectueux de la machinerie traductionnelle, les DRiP (defective ribosomal products) [2].

\section{Les pioneer translation products}

(PTP) : produits d'une traduction précoce des ARN

Même si des études préliminaires suggèrent que les produits de traduction alternatifs dans la cellule sont la source majeure de peptides antigéniques portés par le CMH-I, à ce jour peu de progrès ont été faits sur l'identification des polypeptides dont ils proviennent. Durant le processus de transcription, la particule ribonucléoprotéique qui contient I'ARN - la mRNP (messenger ribonucleoprotein particle) -, se forme [3]. Elle est composée par des complexes protéiques qui s'associent autour de l'ARNm, tels que le complexe d'épissage, mais aussi d'autres facteurs permettant de le protéger d'une éventuelle dégradation. Avant qu'il ne soit permis aux ARNm d'interagir avec des facteurs d'initiation de la traduction (dont elF4E), un mécanisme de contrôle de la qualité de l'ARNm se met en place lors d'un mécanisme de traduction précoce (Figure 1). Nous avons mis en évidence, au cours d'une de nos récentes études, que la synthèse de peptides antigéniques de classe I, à partir d'ARNm codant pour la protéine ovalbumine (Ova), s'arrête bien avant la synthèse de la protéine elle-même, corroborant I'hypothèse des DRiP. De même, nous avons démontré que la production de ces substrats antigéniques coïncide avec une phase précoce de la traduction. Au cours de ces étapes de traduction précoce, appelées pioneer round of translation, les ARNm sont scannés dans le but d'éliminer ceux qui portent des mutations aberrantes. Cependant, nous avons montré que, si un épitope codant pour un peptide antigénique présenté au $\mathrm{CMH}-\mathrm{I}$ est inséré dans un ARNm comportant un codon stop précoce, et qui sera donc dégradé rapidement, cet $A R N m$ est toujours capable de produire des peptides antigéniques en quantité semblable à celle que produirait un ARNm qui ne serait pas dégradé et serait ensuite traduit en une protéine fonctionnelle dans le cytoplasme. Cela suppose que, durant les étapes précoces de la traduction, des polypeptides sont produits, appelés PTP (pour pioneer translation products), qui sont utilisés par la voie du CMH-I afin d'induire une réponse immunitaire. Ce sont les premiers polypeptides produits par les ARNm; ils possèdent les propriétés nécessaires pour être des substrats peptidiques antigéniques, ils sont notamment rapidement dégradées par le protéasome [4] (Figure 1). Jusqu'à récemment, il n'avait pas été possible d'identifier de produits de la traduction d'un ARNm qui puissent être directement liés aux molécules du CMH de classe I et qui permettent ainsi de distinguer des protéines matures fonctionnelles et des produits de traduction alternatifs, source majeure de peptides. Cependant, dans une de nos récentes études, nous avons réussi à purifier, par des techniques d'immuno-affinité et de spectrométrie de masse, les PTP provenant d'un ARNm ne produisant aucune protéine fonctionnelle [5].

\section{Enfin un rôle pour la traduction} nucléaire

Une des nombreuses implications de notre modèle (Figure I) est que la synthèse et/ou la dégradation partielle des polypeptides se ferait dans un compartiment subcellulaire. En effet, les protéines ne sont pas une source majeure de peptides antigéniques pour le $\mathrm{CMH}-\mathrm{I}$. Ainsi, l'induction de la dégradation cytoplasmique par le complexe protéasomique 265 d'une protéine fonctionnelle, 
qui va inonder le cytoplasme cellulaire de produits de dégradation, n'a qu'un effet mineur sur l'approvisionnement des molécules de classe I en peptides. II est donc concevable que les PTP soient dégradés très rapidement après leur synthèse dans un compartiment cellulaire spécifique (encore mal identifié) afin d'éviter que ces polypeptides n'interfèrent de façon non contrôlée avec certaines fonctions cellulaires. Toutefois, le lieu de ce contrôle qualité des ARN néosynthétisés n'est pas encore déterminé avec précision. Une étude récente montre que la seule présence d'un codon stop précoce dans un ARNm donné a pour conséquence de maintenir cet ARNm muté au niveau de la machinerie transcriptionnelle plus longtemps qu'un ARNm non muté. Or, la reconnaissance de ce codon stop précoce par les ribosomes aurait lieu dans le noyau [6]. Cette double observation, d'une part que des ARN en voie de traduction sont scannés par des ribosomes nucléaires, et, d'autre part, que les ARNm portant un codon stop précoce produisent autant de peptides antigéniques que les ARN normaux, suggère qu'un processus de traduction nucléaire - rapporté dans des études anciennes et récentes mais dont la fonction était jusqu'alors inconnue $[7,8]$ - pourrait être la source des PTP. Or, des travaux de recherche en immunologie, portant sur la présentation antigénique de peptides aux molécules de classe I, ont mis en évidence un rôle des ribosomes lors du processus de maturation des ARNm cellulaires. Ainsi, une première observation est celle de peptides antigéniques provenant de séquences introniques $[9,10]$, et une seconde d'une séparation dans l'espace et le temps des processus de production des peptides antigéniques et des protéines fonctionnelles [5]. Le système d'épissage de la cellule fait en sorte que tous les introns d'un ARNm donné soient épissés bien avant que l'ARNm ne quitte le noyau. Par des méthodes de marquage des ARNm, nous avons pu montrer que l'ARNm dans

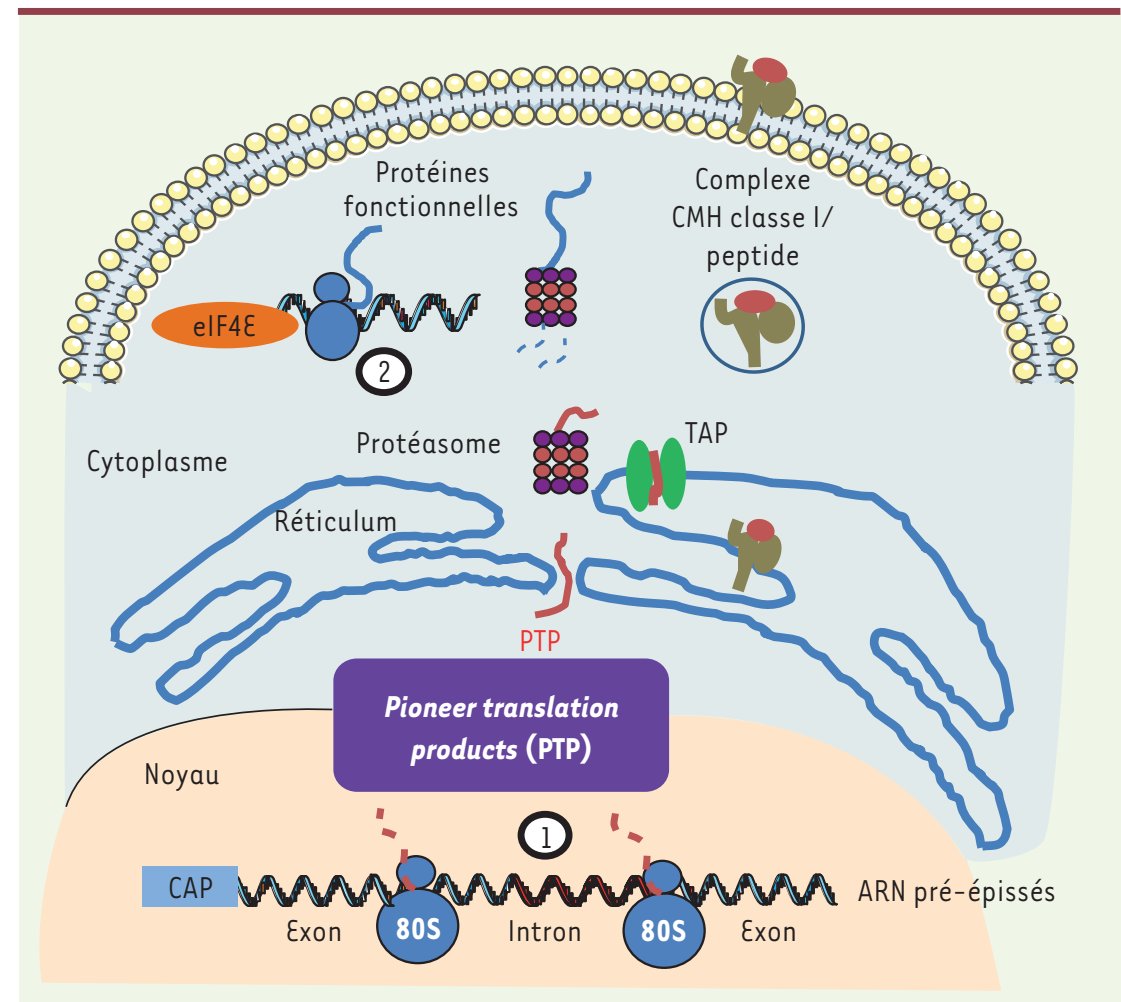

Figure 1. Production des pioneer translation products par les ARN pré-messagers nucléaires. Durant le processus de transcription, la particule ribonucléoprotéique se forme. Elle est composée de l'ARN pré-messager et des complexes protéiques qui s'associent à ce pré-ARNm, tels que le complexe d'épissage, mais aussi d'autres facteurs permettant de le protéger d'une éventuelle dégradation. Cet ARN est alors scanné par des ribosomes nucléaires qui produisent des polypeptides provenant de séquences exoniques mais aussi introniques. Ces polypeptides sont appelés pioneer translation products ou PTP. Ils sont immédiatement dégradés par la voie du protéasome ; les produits de dégradation ont un accès direct au réticulum endoplasmique par le biais du système de transport TAP (transporter associated with antigen presentation) et peuvent ainsi se lier aux molécules de classe I du système immunitaire (étape 1). Une fois que l'ARN pré-messager a été scanné, il peut alors se fixer à d'autres facteurs cytoplasmiques tels que des facteurs d'initiation de la traduction, dont eIF4E, et ainsi commencer à produire des protéines fonctionnelles (étape 2). La dégradation de ces protéines fonctionnelles ne joue qu'un rôle mineur dans la production de peptides antigéniques pour le $\mathrm{CMHI}$, mais pourrait être essentielle pour leur recyclage en acides aminés.

lequel nous avions introduit un épitope antigénique, soit dans un exon, soit dans un intron, est correctement épissé et que seules les séquences exoniques sont détectées dans le cytoplasme, alors que les introns sont confinés dans le noyau [5]. En utilisant la propriété de reconnaissance de cet épitope par des lymphocytes $\mathrm{T} \mathrm{CD}^{+}$spécifiques, nous avons montré très récemment que ce peptide antigénique était présenté à la surface cellulaire, que la séquence ARN correspondante soit d'origine intronique ou exonique [5] (Figure 1). Étant donné que les introns n'atteignent jamais le cytoplasme, les peptides antigéniques correspondants étaient forcément produits par des ribosomes dans le noyau. Nous avons également interféré avec le mécanisme d'export des ARNm du noyau vers le cytoplasme, mécanisme déjà utilisé par certains virus qui exportent directement leurs ARN non épissés vers le cytoplasme. Nous avons pu montrer que si la vitesse d'export des ARN non épissés augmente, la quantité 
de peptides antigéniques diminue en étroite corrélation avec la diminution des ARN nucléaires. Inversement, si I'on diminue l'export nucléaire (par la leptomycine B) dans les cellules qui produisent les PTP, le taux d'ARN dans le noyau augmente, comme attendu, et la production de peptides antigéniques augmente parallèlement [5] (Figure 1). Ces expériences démontrent pour la première fois un rôle de la traduction nucléaire et percent le mystère de la source des peptides antigéniques présentés par le CMH-I. $\diamond$

Synthesis of MHC class I antigenic peptides in the nucleus: a role for the nuclear translation at last?

\section{LIENS D'INTÉRÊT}

Les auteurs déclarent n'avoir aucun lien d'intérêt concernant les données publiées dans cet article.

\section{RéFÉRENCES}

1. Jensen $P E$. Recent advances in antigen processing and presentation. Nat Immunol 2007 ; 8 1041-8.

2. Yewdell JW, Anton LC, Bennink JR. Defective ribosomal products (DRiPs): a major source of antigenic peptides for MHC class I molecules? I Immunol 1996; $157: 1823-6$

3. Muller-McNicoll M, Neugebauer KM. How cells get the message: dynamic assembly and function of mRNA-protein complexes. Nat Rev Genet 2013 ; 14 : 275-87.

4. Apcher S, Daskalogianni C, Lejeune F, et al. Major source of antigenic peptides for the MHC class I pathway is produced during the pioneer round of mRNA translation. Proc Natl Acad Sci USA $2011 ; 108$ 11572-7.
5. Apcher S, Millot G, Daskalogianni C, et al. Translation of pre-spliced RNAs in the nuclear compartment generates peptides for the MHC class I pathway. Proc Natl Acad Sci USA 2013 ; 110 : 17951-6.

6. de Turris V, Nicholson P, Orozco RZ, et al. Cotranscriptional effect of a premature termination codon revealed by live-cell imaging. RNA $2011 ; 17$ : 2094-107.

7. Iborra FJ, Jackson DA, Cook PR. Coupled transcription and translation within nuclei of mammalian cells. Science 2001 ; 293 : 1139-42.

8. David A, Dolan BP, Hickman HD, et al. Nuclear translation visualized by ribosome-bound nascent chain puromycylation. J Cell Biol 2012 ; 197 : 45- 57.

9. Guilloux $Y$, Lucas $S$, Brichard VG, et al. A peptide recognized by human cytolytic T lymphocytes on HLAA2 melanomas is encoded by an intron sequence of the $\mathrm{N}$-acetylglucosaminyltransferase $\mathrm{V}$ gene. J Exp Med 1996; 183 : 1173-83.

10. Coulie PG, Lehmann F, Lethé $B$, et al. A mutated intron sequence codes for an antigenic peptide recognized by cytolytic T lymphocytes on a human melanoma. Proc Natl Acad Sci USA 1995 ; 92 : 7976-80.

\section{NOUVELLE}

\section{IAP et Rho : enfin connectées}

\author{
Laurence Dubrez, Arthur Marivin, Jean Berthelet
}

Inserm UMR866, Faculté de médecine,

7 boulevard Jeanne d'Arc, 21079 Dijon,

France ; Université de Bourgogne,

21079 Dijon, France.

Idubrez@u-bourgogne.fr

Rho [4]. Les mécanismes moléculaires n'ont pas été clairement établis, cependant, les auteurs ont mis en évidence une interaction de DIAPl avec Rac.

\section{La famille des protéines $R$ ho}

La famille Rho comprend 22 membres dont les chefs de files sont RhoA, Racl et cdc42. Ce sont des régulateurs importants des modifications du cytosquelette d'actine [5]. Ces molécules contrôlent ainsi tous les changements morphologiques de la cellule, son adhésion, sa migration, sa polarisation, ainsi que les transports vésiculaires intracellulaires. Ce sont des GTPases, majoritairement présentes dans le cytosol, dans une conformation inactive liée au nucléotide guanylique GDP (guanosine-5'-triphosphate). En réponse à différents stimulus, elles adoptent une conformation active liée au GTP (guanosine-5'-triphosphate), qui leur permet d'activer leurs effecteurs (Figure 1). Les membres de la famille Rho ont des fonctions 